\title{
Deep total intravenous anesthesia reduced the incidence of early but not long-term cognitive dysfunction in the elderly
}

\author{
Chang Liu,M.D.,Qulian Guo, M.D. \\ Anesthesiology, Xiangya Hospital of Central-South University, Changsha, China.
}

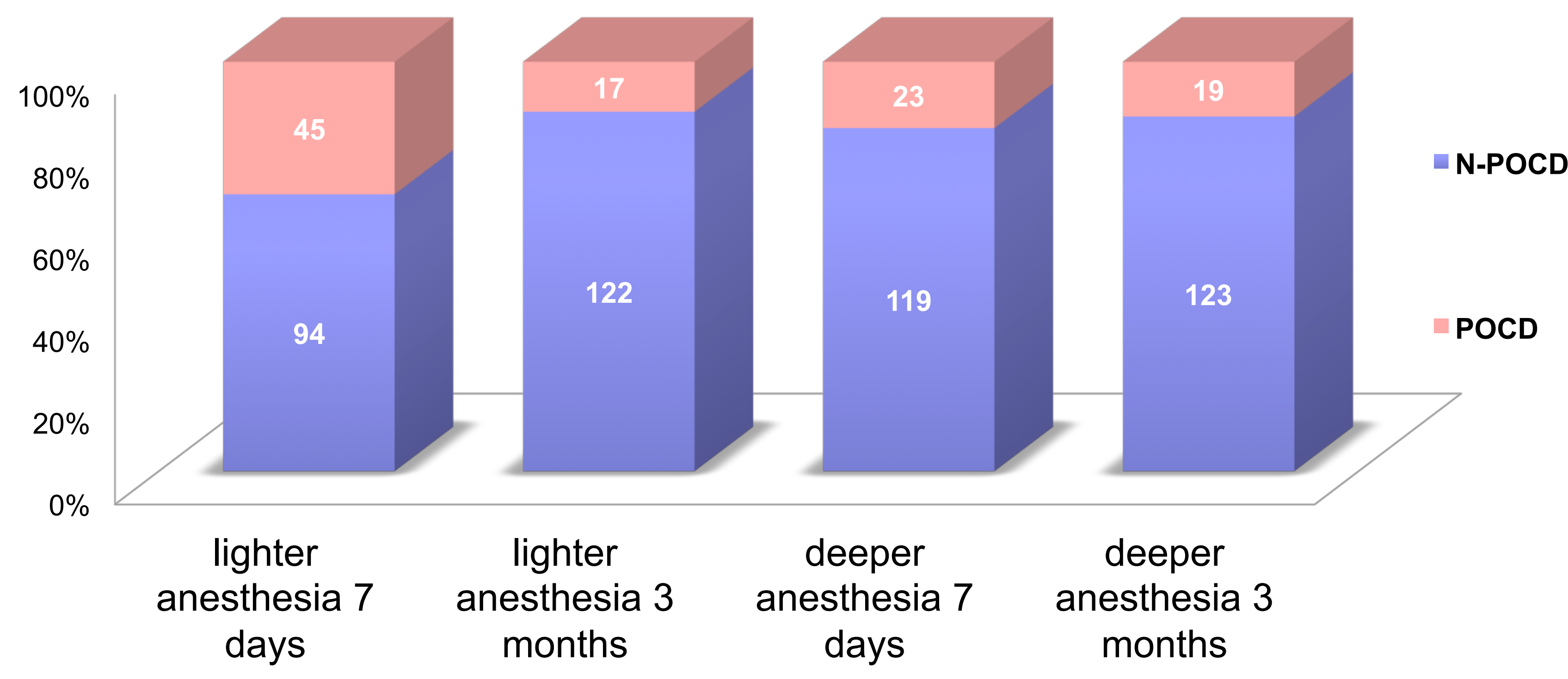

Background and Goal of Study: Cognitive dysfunction is common in adult patients and the elderly are at significant risk for long-term cognitive problems. Depth of anesthesia has also been reported to influence POCD. But up to now, the effect of the depth of anesthesia on POCD remains controversial. This study is designed to investigate whether the depth of anesthesia affects the incidence of POCD, we will use propofol for total intravenous anesthesia in elderly patients whose depth of anesthesia was monitored and adjusted by bispectral index (BIS). The early(7days) and long-term(3 months) incidence of POCD will be compared between deep and light-anesthesia groups.

Methods: 357 patients aging between 50 and 70 were randomly divided into 2 groups: deeper anesthesia $(n=181)$ and lighter anesthesia $(n=176)$. Elderly patients who are going to receive moderate laparotomic operations for the first time are selected (including gastrectomy, gastric cancer, colon resection, urinary calculi types of surgery, hysteromyomectomy). The operative time is expected to be in 90-150 min, and the amount of bleeding is expected to be less than $500 \mathrm{ml}$ ).Exclusion criteria included: endoscopic surgery using carbon dioxide, history of neurologic or mental disease, renal dysfunction serum creatinine in excess of $177 \mathrm{mmol} / \mathrm{L}$, active liver disease, cardiac dysfunction, pulmonary dysfunction, endocrine disease, metabolic disease, a history of surgery, fewer than 6 years of education, inability to complete neuropsychologic testing, vision dysfunction, and auditory dysfunction. Total intravenous anesthesia was used. Propofol and sufentanil were used for anesthesia induction and propofol and remifentanil infusion rate titrated to maintain target BIS values.
BIS is maintained in 35-45 in deep anesthesia group and 50-60 in light anesthesia group. A battery of 9 neuropsychologic tests (cumulative learning, verbal fluency, association test, digit span(forward and backward), digit symbol, trail making test, grooved pegboard test(favored and non-favored hand)) was administered preoperatively, 7days and 3 months after surgery. A postoperative deficit was defined as a postoperative decrement to preoperative score greater than I standard deviation on any test. Patients who experienced 2 or more deficits were deemed to have early postoperative cognitive dysfunction.

Results: 28I patients completed both preoperative and postoperative neuropsychologic testing, of which 142 were in deeper and 139 in the lighter anesthesia group. The early(7days) postoperative cognitive dysfunction occurred in 23 patients (16.2\%) in the deeper anesthesia group and in 45 patients $(32.3 \%)$ in the lighter anesthesia group. The longterm(3 months) postoperative cognitive dysfunction occurred in 19 patients $(13.3 \%)$ in the deeper anesthesia group and in 17 patients (12.2\%) in the lighter anesthesia group. The difference in incidence of cognitive dysfunction between deeper anesthesia group and lighter anesthesia group is statistically significant $(P=0.0015, \chi 2)$ at 7 days but not at 3 months $(P=0.7527, \chi 2)$.

Conclusion: Deeper total intravenous anesthesia can decrease the incidence of cognitive dysfunction in the early(7days) postoperative period but had no significant effect on long-term(3 months) cognitive dysfunction.

Key Words: bispectral index (BIS), depth of anesthesia, postoperative cognitive dysfunction (POCD), total intravenous anesthesia 